\title{
EVALUACIÓN PARTICIPATIVA EN HABILIDADES PARA COMUNICAR EN 3 DE GRADO DE ENFERMERÍA EN EL CURSO 2009/10, LA ESCENIFICACIÓN COMO MÉTODO DOCENTE Y DE EVALUACIÓN.
}

\author{
L'ÉVALUATION PARTICIPATIVE DANS LA MATIÈRE COMPÉTENCES POUR LA \\ COMMUNICATION DANS 3 DU DEGRÉ D'INFIRMERIE DANS LE COURS 2009/10, \\ LE ROL PLAYING COMME UN MÉTHODE D’ENSEIGNANTE ET ÉVALUATION.
}

\begin{abstract}
PARTICIPATIVE EVALUATION IN COMMUNICATION SKILLS IN THIRD YEAR OF NURSING DEGREE, 2009/ 2010, ROL PLAYING AS A TEACHING AND EVALUATION METHOD.
\end{abstract}

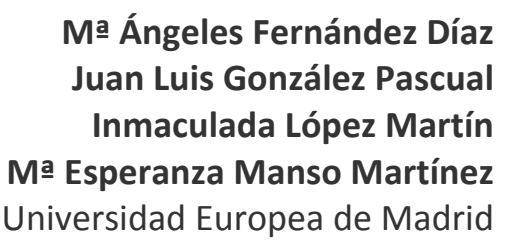

\section{RESUMEN}

La materia "Habilidades para Comunicar y Educar en Salud" incluye la comprensión de la enfermería como una relación humana cliente - enfermero/a para identificar y responder a la necesidad de ayuda, desarrollando la comunicación como principal instrumento para la prestación de cuidados.

Para alcanzar las competencias en esta asignatura, durante el curso 2009-10, se desarrolló una metodología activa basada en un aprendizaje significativo. La evaluación se realizó a través de la demostración práctica de la adquisición de habilidades en comunicación verbal y no verbal, mediante técnicas de dramatización evaluadas por el profesorado y complementada por una evaluación entre iguales. Este sistema supuso un desafío tanto para estudiantes como para profesores.

Con objeto de conocer: grado de acuerdo del alumnado con esta metodología de aprendizaje y evaluación, concordancia de resultados en evaluaciones de profesorado - alumnado y nivel de consecución de los objetivos de aprendizaje respecto al bloque temático de Comunicación y Relación de Ayuda, se diseñó un estudio descriptivo transversal con 95 alumnos/as. Los resultados mostraron la satisfacción de los alumnos/as, la falta de concordancia en las evaluaciones de profesorado y alumnado (coincidiendo con otros estudios) y evidenciaron la eficacia de esta metodología para adquirir habilidades específicas en comunicación.

\section{PALABRAS CLAVE}

Evaluación participativa, rol playing, escenificación, habilidades para comunicar, aprendizaje significativo. 


\begin{abstract}
"Communication and Education Skills for Health" includes an understanding of nursing as a human relation client - nurse to identify and respond to the need of help, developing communication as the main instrument for the provision of care. To achieve competence in this subject during the academic course 2009-10, we developed an active methodology based in meaningful learning. The evaluation was made through practical demonstration of the acquisition of skills in verbal and nonverbal communication through drama techniques (rol-playing) evaluated by teachers and supplemented by a peer review. This system was a challenge for both students and teachers.

In order to know: the agreement of students with this method of learning and assessment, the agreement of results between teacher and student's assessments and the level of achievement of learning objectives in the unit "Helping Relationship and Communication", we designed a descriptive study with 95 students. The results showed the satisfaction of the students, the lack of consistency in the evaluations of teachers and students (coinciding with other studies) and demonstrated the effectiveness of this methodology to acquire specific skills in communication.
\end{abstract}

\title{
KEY WORDS
}

Participative evaluation, rol playing, staging, communication skills, meaningful learning.

\section{RÉSUMÉ}

La matière "Compétences pour la communication et l'éducation pour la santé" comprende une compréhension des soins infirmiers comme une relation client infirmièr/e pour identifier et répondre aux besoins d'aider à développer la communication comme instrument principal de la prestation de soins.

Pour atteindre les compétences dans cette matière au cours de l'année académique 2009-10, nous avons développé une méthodologie active baseé sur un apprentissage significatif. L'évaluation a été réalisée par des démonstrations pratiques de l'acquisition d'aptitudes en communication verbale et non verbale grâce à des techniques théâtrales évalués par les professeurs et complétées par les pairs. Ce système a representé un défi pour les étudiants et les enseignants.

Nos objetifs ont été: connaître le degré d'acceptation de cette méthode d'apprentissage et d'évaluation para les étudiants, connaître la concordance de l'évaluation des enseignants et de celle des élèves, en plus du niveau d'atteinte des objectifs d'apprentissage de l'unité thématique "Communication et Relations d'aide". Nous avons réalisé une étude descriptive avec 95 étudiants. Les résultats ont montré la satisfaction des étudiants, ont révélé un décalage entre les évaluations des enseignants et celles des étudiants (confirmées par d'autres études) et ont démontré l'efficacité de cette méthode pour acquérir des compétences spécifiques en matière de communication.

\section{MOTS CLEFS}

Évaluation participative, rol playing, mise en scène, habilité pour communiquer, apprentissage significatif. 


\section{INTRODUCCIÓN}

Estamos en la era de la información, la cantidad de información a la que tenemos acceso se puede decir que es infinita, cambiante y dinámica. La información se intercambia rápidamente y el conocimiento crece de manera exponencial. No parece por tanto posible para un docente, hoy en día, llegar a dominar todo el conocimiento que comprende su campo o disciplina, sino saber gestionarla y desarrollar en los estudiantes las habilidades necesarias para "navegar" en los océanos del saber e invertir en desarrollar capacidades para aplicarlas en el mundo profesional. El profesor ahora se representa más bien como una llave para abrir la puerta de dominio del conocimiento y la experiencia (Dochy, 2002).

\section{EL PAPEL DE LA COMUNICACIÓN EN LA ADQUISICIÓN DE COMPETENCIAS}

La formación en competencias implica el paso de un modelo pedagógico de base conductista a enfoques de base constructivista (Fernández, 2005), en consonancia con la aplicación de estrategias metodológicas de construcción del conocimiento desarrolladas por los estudiantes.

De la aplicación de dichos métodos surgen tareas que promueven el desarrollo de capacidades de análisis, síntesis, juicios críticos y toma de decisiones, de las cuales surge conocimiento nuevo. Uno de los componentes que caracteriza a estas capacidades es que integra lo que ya se sabe, se piensa y se siente, con la construcción de significados nuevos en un marco de colaboración y comunicación con otros sujetos (López, González y Agudo, 2007). Durante décadas, el objetivo principal de la formación académica ha sido formar estudiantes con muchos conocimientos dentro de una determinada área. La tarea consistía en construir una colección de conocimientos básicos. Sin embargo, actualmente se hace énfasis en la formación de individuos con un alto nivel de conocimientos, pero también con habilidades para resolver problemas, habilidades profesionales y aprendizaje "auténtico", entendido como aprendizaje en contextos de la vida real. El mundo actual necesita ciudadanos autónomos, adaptables, pensantes, que aprendan de forma autónoma, capaces de comunicarse y cooperar con otros (Dochy, 2002).

La construcción de nuevos significados surge en el contexto de un aprendizaje de tipo dialógico, no necesariamente en un entorno académico como es la universidad. En todas las dimensiones o esferas de la educación está presente la comunicación a través de la interrelación personal, como medio para enseñar y aprender; que es en definitiva el objetivo final de la educación. La adquisición de competencias cognitivas depende en gran medida de la relación con otros, profesores, tutores, estudiantes. La capacidad para la resolución de problemas, el desarrollo del pensamiento crítico, la formulación de preguntas, búsqueda de información relevante, elaboración de juicios informados, uso eficiente de la información, realizar observaciones e investigaciones precisas, inventar y crear cosas nuevas, analizar datos, etc. Tienen una relación 
estrecha con los métodos de enseñanza-aprendizaje y el desarrollo de habilidades comunicativas para presentar los datos, expresarse de forma oral y escrita.

El trabajo en equipo, competencia transversal destacada en los planes de estudios actuales de las titulaciones universitarias de Grado, se aprende practicándola por medio de discusiones y conversaciones, cooperación, trabajo en grupos, etc.,

En la conversación y en la reflexión que se produce durante la interrelación personal, es donde se da el aprendizaje llamado dialógico. A través de la palabra, lo paraverbal y lo no verbal, intercambiamos, modificamos y creamos significados, y nos ponemos de acuerdo sobre ellos (Flecha, 2002:21).

Habermas (1987, citado por Flecha, 2002), en su teoría de la competencia comunicativa, distingue cuatro tipos de acciones diferentes, en lo que se refiere a la acción comunicativa basada en un diálogo entre iguales, las personas aprenden por medio de la interrelación establecida y desde las necesidades, demandas y prioridades del que desea aprender. Se aprende mediante el diálogo y la práctica de la conversación, aprendiendo sobre la propia comunicación.

La metacomunicación consiste en hablar sobre cómo nos comunicamos, someter a análisis la propia comunicación (Zabalza, 2007) y aprehenderla como un valor intrínseco en nuestras vidas, a nivel personal, familiar y profesional. De forma que profesores y estudiantes, cuando hablan de la comunicación, abordan la importancia de las relaciones y de qué forma pueden ser revisadas, modificadas desde la comprensión de estas, y utilizadas como un recurso para cuidar de sí y de otros.

\section{LAS HABILIDADES COMUNICATIVAS SE ENSEÑAN Y SE APRENDEN}

Partiendo de la debida coherencia y consonancia entre los componentes que se estructuran en los sistemas formativos universitarios: objetivos formativos, contenidos actualizados, manejo de metodologías y recursos actualizados, etc., es el bloque de evaluación el que tiene una relevancia especial. Constituye la parte de nuestra actividad docente, que tiene más fuertes repercusiones sobre los alumnos y alumnas (Zabalza, 2007). Consecuencias tangibles en cuanto a resultados académicos, repercusiones administrativas, etc. $Y$ no tan tangibles, como los efectos de la evaluación sobre la autoestima, motivación hacia el aprendizaje, significado punitivo o formativo, meta o recurso. Cuando está comenzando una materia, al alumno/a le interesa especialmente el sistema de evaluación, el cómo se le va a evaluar, el peso específico de cada prueba, las condiciones de realización de estas.

Las investigaciones sugieren que las habilidades comunicativas no mejoran necesariamente sólo con la experiencia, sino con la formación. Se están destinando considerables esfuerzos y recursos para desarrollarlas, porque dichas habilidades se pueden enseñar (Fallowfield 1990; Fallowfield 2001 y otros citado por Fellowes, 2008) y mantener con el transcurso del tiempo. Profesionales de la salud especialmente asisten a cursos y talleres de habilidades comunicativas para mejorar la atención a sus pacientes, con el objetivo especifico de fomentar un mejor conocimiento de las preocupaciones y sentimientos del usuario del sistema sanitario y posibilitar una 
atención óptima (Fellowes, 2008). La mayoría de los enfoques de enseñanza incorporan componentes cognitivos, afectivos y conductuales, y se centran en el sujeto que está aprendiendo, en el sentido de "llevar las riendas" del diálogo o entrevista en un marco de atención clínica.

Qué duda cabe que una buena comunicación entre los profesionales de la salud y los clientes es esencial para proporcionar una atención de calidad. Diversas investigaciones citadas por Cléries y Borrell (2003), indican que para los pacientes, uno de los atributos fundamentales del personal sanitario es la capacidad para escuchar y explicar, principios necesarios para poder hacer "feedback" y que se produzca un acto de comunicación eficaz. Cuestión importante para la valoración de problemas de salud, el cumplimiento de regímenes terapéuticos, la motivación para realizar cambios de comportamientos en salud y sentirse comprendido respecto a los avatares en la vida de las personas. Y estamos hablando de "empatía".

Habitualmente, las quejas presentadas por las personas en las instituciones sanitarias, tienen que ver mucho más con la percepción de falta de comunicación que con una falta de competencia clínica. En algún momento de nuestras vidas, todos y cada uno de nosotros ha sentido que el profesional que nos atendía no era capaz de transmitir de manera adecuada cualidades como el interés sincero por el motivo de consulta, la escucha sin condicionantes, etc.

La incorporación de habilidades de comunicación en las competencias de las profesiones sanitarias queda justificado, según Cléries, Borrell y otros (2003), por diversos factores: el progreso de la sociedad y la toma de conciencia por parte del ciudadano de su mayor implicación participativa en el cuidado de su salud y por lo tanto en la toma de decisiones respecto a esta. Por otra parte el cliente es muchas veces, experto en alguna área de conocimientos. En segundo lugar la percepción de pérdida de prestigio por parte de los profesionales sanitarios, debida en parte a no saber comunicarse con el paciente y con la sociedad y al incremento del grado de cultura en general que conlleva la globalización.

\section{LA COMUNICACIÓN EN LA DISCIPLINA ENFERMERA}

La enfermería es una relación humana entre un cliente y una enfermera que posee una formación especializada, para reconocer y responder a la necesidad de ayuda; de esta relación surge frecuentemente, el esclarecimiento del problema del paciente (Mejía, 2006). Para que las enfermeras realicen cuidados, siendo este su objeto de conocimiento y razón de ser, se requiere una formación científica, tecnológica y humanística que le permita, en su interacción con los pacientes, realizar acciones que exigen la participación de ambos.

El principal instrumento para la prestación de cuidados es la comunicación establecida entre enfermera y cliente/s. Y a su vez, esta es el principio fundamental para la realización de educación para la salud. La Organización Mundial de la Salud (OMS) definió en 1983 la Educación para la Salud (EpS) como cualquier combinación de actividades informativas y educativas que llevan a una situación en la que la gente desee vivir sana, sepa cómo alcanzar la salud, haga lo que pueda individual y colectivamente para mantenerse en salud y busque ayuda cuando la necesite. 
La experiencia de comunicar impregna cualquier acción relacionada el ejercicio de la atención sanitaria. Constituye lo ontológico o el centro en la epistemología de Enfermería. Los modelos y teorías enfermeras que giran en torno al cuidado de las personas, familias y otras agrupaciones de individuos en la comunidad, contemplan la comunicación como el eje alrededor del cual se articular las diferentes formas de entender el cuidado.

Los desarrollos de la enfermería, como una ciencia eminentemente social, han surgido justamente de las interacciones personales entre enfermeras y pacientes; cada una de las teóricas de la disciplina enfermera ha trascendido esta relación por diversos intereses. Para Virginia Henderson, esta relación surge en la medida en que la enfermera es capaz de identificar y satisfacer las 14 necesidades del ser humano. La enfermera es suplente o es ayuda en la satisfacción de dichas necesidades, mientras el paciente se prepara para hacerlo por sí mismo. Su objetivo es hacer al individuo independiente lo antes posible (Marriner, 2006).

Para Ernestine Widdenbach, las relaciones interpersonales entre paciente y enfermera son posibles cuando ésta interpreta la necesidad de ayuda del paciente y para ésto son importantes los conocimientos tanto de biología como de sociología. La persona debe mantener su equilibrio fisiológico y emocional puesto que el cuerpo y la mente son inseparables, requiere ayuda para conseguir su independencia.

Madeleine Leininger, en su teoría de la diversidad y universalidad de los cuidados culturales, presenta su convicción de que las personas de culturas diferentes pueden ofrecer información y orientar a los profesionales para recibir los cuidados que desean o necesitan de los demás. La cultura determina los patrones y estilos de vida que influyen en las decisiones de las personas, para ayudar a la enfermera a descubrir y documentar el mundo del paciente (Marriner, 2006).

A Hildegard E. Peplau le interesa, principalmente, delinear las tareas psicológicas que se desarrollan en situaciones interpersonales, particularmente la relación enfermera-paciente. El alcance de la enfermería depende de la capacidad de la enfermera para reconocer las dificultades que plantean las relaciones con las personas y de la habilidad que logre para ayudarlas a resolver sus dificultades, de tal manera que puedan desarrollar nuevas facultades para afrontar problemas recurrentes; al llegar a este punto, ella considera que el paciente está maduro y es capaz de comprender su situación.

Peplau describe y analiza cuatro fases en la relación enfermera - paciente: en la fase de orientación, el paciente busca ayuda como consecuencia de su enfermedad y en la interacción con la enfermera, puede encontrar alternativas para la adaptación. En la fase de identificación, el paciente cree conocer lo que le ofrece la situación y responde selectivamente a quienes le brindan ayuda. En la fase de explotación, intenta de diversas formas sacar partido a la relación y explotar los bienes y servicios existentes a su disposición. En la fase de resolución, tan pronto como se satisfacen por completo las antiguas necesidades, el paciente las pospone gradualmente (Mejía, 2006). 
Los conocimientos de enfermería deben conducir el cuidado hacia la solución de problemas; pero para ello, la enfermera requiere habilidad en la identificación de éstos, destreza que se demuestra en poder ir más allá de la historia clínica, de los medicamentos, de los monitores y de los tubos que rodean al paciente (Mejía, 2006).

\section{EL APRENDIZAJE Y LA EVALUACIÓN DE LAS HABILIDADES EN LA COMUNICACIÓN}

La necesidad de integrar las habilidades de comunicación en las enseñanzas de ciencias de la salud es vox populi desde hace décadas, de forma transversal y sin perjuicio de que existan créditos específicos sobre la materia. Esto se traduce en la introducción de conocimientos y habilidades específicas y centradas en el trato con las personas y en basar la relación sanitario-paciente en una correcta comunicación terapéutica; de forma que la docencia en habilidades para comunicar esté presente en todos los niveles educativos y especialmente en las titulaciones de Grado (Cláries, Borrell, 2003). Somos también de la opinión de que las habilidades sociales y de comunicación deben seguir abordándose como formación profesional continuada.

La evaluación de habilidades de comunicación debe realizarse de forma directa, con metodologías que aúnen el trabajo con casos y situaciones lo más reales posibles, creando escenarios y personajes cercanos a la realidad y con la utilización de recursos teatrales que le aporten veracidad, en un marco de práctica reflexiva, análisis del discurso, aprendizaje significativo y metacomunicación (ver figura 1).

La coevaluación, evaluación colaborativa entre alumnos y profesores, y la evaluación entre iguales o por pares (Brawn y Glasner, 2007; Dochy, 2006) son estrategias de evaluación participativa y formativa, con numerosos beneficios descritos en la bibliografía educativa e implica una concepción más democrática del proceso de evaluación.

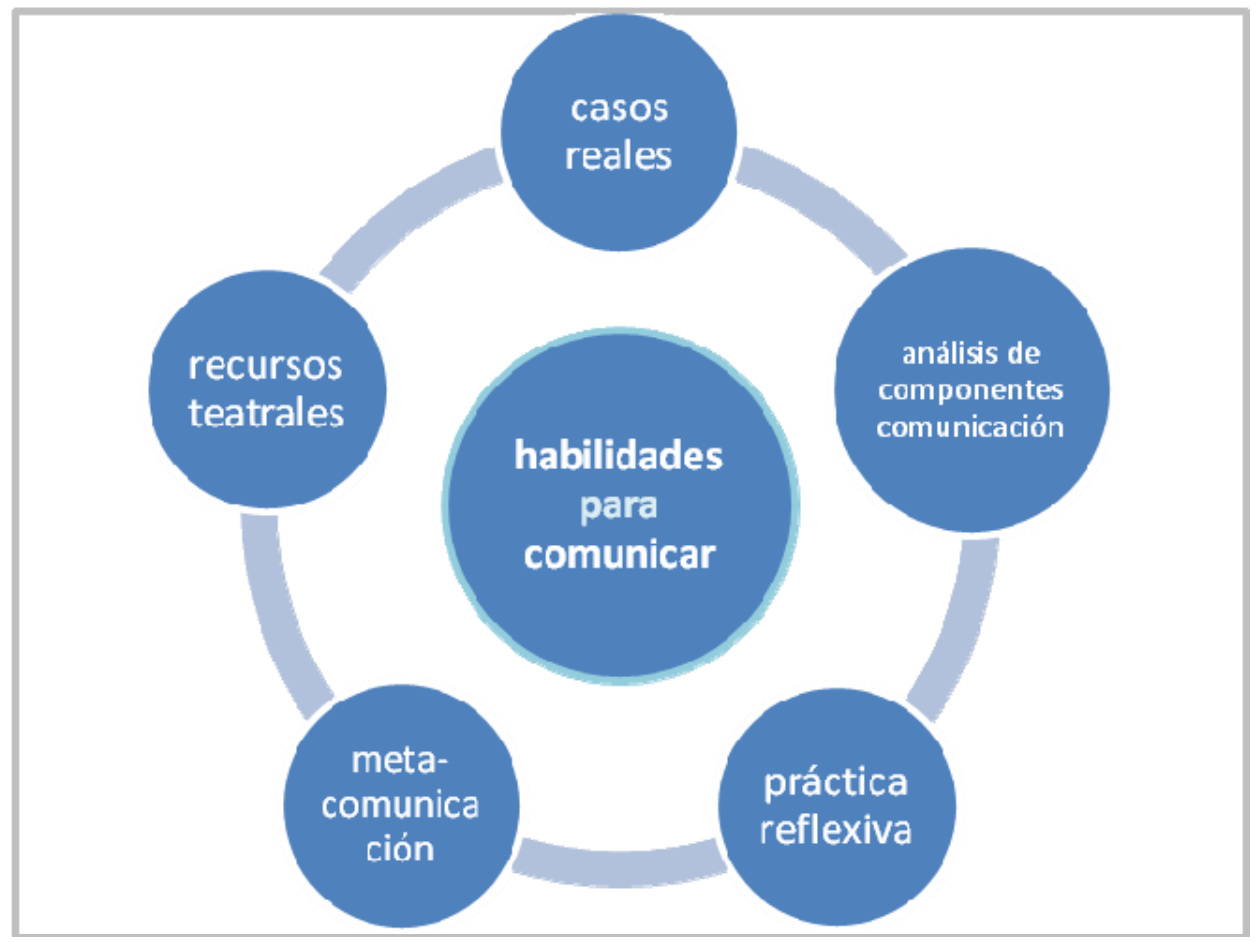

Figura 1.- Evaluar Habilidades de Comunicación: Conjunción de Estrategias y Métodos 


\section{EL ROL-PLAYING COMO ESTRATEGIA DE ENSEÑANZA- APRENDIZAJE Y EVALUACIÓN}

Dice Lowen (citado por Caballer y cols, 2005) que el ser humano adulto tiene la misma necesidad de autoexpresión que los niños. Los niños expresan mediante el juego y de forma simbólica sus emociones y sus necesidades. Los adultos, cuando encuentran un espacio libre para la expresión, se implican de igual manera aunque con mayor esfuerzo a la participación en juegos de imaginación con el fin de expresar ideas y suspender la realidad exterior (Caballer y cols, 2005). El rol-playing, como técnica de desarrollo de habilidades es un ejemplo de espacio para la expresión, emulación de situaciones reales susceptibles de analizar y modificar en un plano imaginativo o virtual, transferible a la realidad en la práctica asistencial.

El rol-playing trabaja las habilidades sociales a un nivel más profundo que la simulación operativa. Su objetivo es desarrollar habilidades concretas el área de las aptitudes, psicomotoras, personales y sociales (pág. 88 del Manual de Educación para la Salud del Gobierno de Navarra, 2006). La escenificación de situaciones reales o ficticias es el instrumento para aprender y demostrar conocimientos enfermeros aplicables en dicha situación, la aplicación y adaptación de esquemas de comunicación y manejo intencionado de técnicas terapéuticas en la relación de ayuda.

El procedimiento general consiste en informar en primer lugar del contexto en el que se produce la escenificación (guión escrito), definir el perfil básico de los actores y definir el papel de los observadores (alumnos no actores) discentes y del observador docente. Aclarar cuál es el objetivo de la escenificación y en qué aspectos o ítems debemos fijar la atención durante su realización. Por último se realiza una puesta en común sobre lo observado respecto al modelo teórico y su aplicación real.

\section{LA MATERIA “HABILIDADES PARA COMUNICAR Y EDUCAR EN SALUD”. 3 DE GRADO DE ENFERMERIA}

La materia "Habilidades para Comunicar y Educar en Salud", impartida por primera vez en la Universidad Europea de Madrid en el curso académico 2009/10 en 3으 de Grado en Enfermería, tiene como principal objetivo el desarrollo de habilidades y destrezas en los estudiantes de Grado, en el proceso de comunicación con los pacientes, sea cual sea el ámbito de actuación de la enfermería, y la capacitación para informar, asesorar y educar en situaciones de salud, de riesgo de enfermar, en situaciones de dependencia y al final de la vida.

Para conseguir su objetivo, tiene una orientación transversal; de forma que proporcione cimientos en cuanto a la comunicación eficaz y a contenidos de Educación para la Salud (EpS) para la atención enfermera en diferentes niveles de atención, a individuos y grupos, institucionalizados o viviendo en su entorno habitual; en diferentes momentos del continuo salud-enfermedad y en las diferentes etapas del ciclo vital de las personas.

En Habilidades para comunicar y educar en salud está implícito un concepto que se considera importante en la adquisición de competencias en un alumno/a de 3 으 de Grado y de cara al 4 o y último curso:

La enseñanza-aprendizaje de los conocimientos y habilidades para comunicar y educar en salud deben basarse en métodos docentes que promuevan la participación y 
las destrezas; como por ejemplo la demostración, técnicas de dramatización, la expresión oral y la aplicación de medios audio-visuales, entre otras. Estos métodos y medios van a guiar el desarrollo de la materia y también el sistema de evaluación propuesto a los estudiantes.

Competencias especificas propuestas en la materia, en el bloque de Habilidades para Comunicar:

1. Establecer una comunicación eficaz con pacientes, familia, grupos sociales y compañeros y fomentar la educación para la salud.

2. Comprender el comportamiento interactivo de la persona, objeto de atención enfermera, en función de su estado de salud, del género, grupo o comunidad, dentro de su contexto social y multicultural.

3. Ser capaz de comunicar de forma oral y escrita contenidos de EpS.

Objetivos de aprendizaje específicos respecto al bloque de Habilidades para Comunicar, para desarrollar las competencias enunciadas:

- Desarrollar habilidades en la comunicación con individuos, familias y grupos.

- Identificar las etapas de la relación de ayuda durante las entrevistas simuladas a pacientes y/o familias.

Contenidos del programa: Bloque de Comunicación y Relación de Ayuda:

- Fundamentos y bases teóricas de la comunicación humana.

- El papel de la comunicación desde el Marco teórico de Enfermería.

- La relación de ayuda con individuos, familias y grupos.

- Herramientas de comunicación efectiva.

- Enfoques específicos de comunicación en el proceso de atención de enfermería. Utilización de cuestionarios, escalas y test en la entrevista de valoración estructurada.

- Aplicación/adecuación de la relación de ayuda y técnicas de comunicación según etapa vital, nivel de atención y en situaciones de riesgo o complejas.

Metodologías docentes utilizadas en la materia: Exposiciones del profesor/a, Técnicas de desarrollo de habilidades: rol-playing (ver fig. 2) y Trabajo colaborativo en pequeño grupo. 
Tema del Rol-Playing y correspondencia con contenidos del temario.

Objetivos de aprendizaje a través del Rol-Playing.

Contextualización de la escena y Definición de personajes.

Ejecución.

Preguntas del profesor a actores y observadores.

Puesta en común. Feed-back.

Conclusiones y cierre

Figura 2.- Esquema general de planificación del rol-playing en la materia Habilidades para Comunicar y Educar en Salud.-

Los aspectos que formaron parte del sistema de evaluación en el bloque de Comunicación y Relación de Ayuda fueron:

- Asistencia y participación activa en el aula, es decir, la observación y realización de los diferentes rol-playing puestos en práctica como método de enseñanzaaprendizaje (15\% respecto a la nota global).

- Realización de una escenificación recreada (adecuación del espacio, uso de material clínico, etc.) en sala de demostración/simulación (30\% respecto a la nota global). El estudiante adopta el papel de profesional de enfermería, y dos profesores adoptan los roles de observador-evaluador y persona que recibe los cuidados respectivamente.

El instrumento de evaluación fue una rúbrica diseñada a propósito, con los ítems y escala para estimar el grado de consecución de los objetivos y competencias, mediante la coevaluación y evaluación entre iguales (ver anexo).

Al finalizar la impartición de la materia y ante lo innovador de la misma y de su sistema de evaluación, tomamos la decisión de analizar en profundidad los resultados cuantitativos (rendimiento académico), así como las opiniones de estudiantes y profesores respecto a la experiencia docente, novedosa para todos los participantes. Por ello nos proponemos como objetivos de investigación, los siguientes:

- Evaluar el grado de acuerdo del alumnado, respecto al diseño, metodología y forma de evaluación de la materia Habilidades para Comunicar y Educar en Salud, específicamente en el bloque temático de Comunicación y Relación de Ayuda, en el curso 2009/10.

- Evaluar la concordancia en los resultados de evaluación entre profesores y alumnado, respecto al bloque temático de Comunicación y Relación de Ayuda, curso 2009/10. 
- Establecer el grado de consecución de los objetivos de aprendizaje planteados en los alumnos/as, respecto al bloque temático de Comunicación y Relación de Ayuda, curso 2009/10.

\section{MÉTODOS E INSTRUMENTOS:}

Estudio descriptivo transversal.

Población: 175 estudiantes de 3 curso de Grado en enfermería matriculados en la materia Habilidades para Comunicar y Educar en Salud, en turno de mañana, del curso académico 2009/10.

\section{Muestra: 95 estudiantes}

Criterios de selección de la muestra: A partir del total de los estudiantes matriculados, se ha excluido a los que no se presentaron a la prueba de evaluación y a los que no cumplimentaron correctamente los ítems de evaluación de la rúbrica (ver anexo).

Se pidió a los estudiantes que hicieran una valoración en una escala de 1 a 6 ( 1 total desacuerdo, 6 totalmente de acuerdo) acerca del método de evaluación. Además, el profesorado recogió comentarios de los estudiantes referidos al proceso de evaluación.

A partir de la puntuación de los ítems de evaluación de la rúbrica, se ha calculado la mediana y el rango intercuartil para la puntuación total y para la puntuación parcial de cada uno de los bloques (Componente no verbal, Componente paraverbal, Relación de ayuda y Técnicas de comunicación), tanto para la calificación otorgada por los estudiantes como la otorgada por el docente.

Para valorar la concordancia entre la puntuación de estudiantes y docente, se ha calculado el Coeficiente de Correlación Intraclase (CCI) con su correspondiente intervalo de confianza al 95\% (IC 95\%).

Además, se ha calculado la mediana y el rango intercuartil para la puntuación otorgada por los estudiantes a su grado de acuerdo con el método de evaluación.

\section{RESULTADOS}

La mediana del grado de acuerdo del alumnado respecto al diseño, metodología y forma de evaluación de la materia, en la escala de 1 a 6 , fue de 5 (amplitud intercuartil 4 a 5).

\begin{tabular}{|cc|}
\hline Puntuación & Porcentaje \\
\hline 1 & $1 \%$ \\
\hline 2 & $3,5 \%$ \\
3 & $5,3 \%$ \\
4 & $28,3 \%$ \\
5 & $45,1 \%$ \\
6 & $16,8 \%$ \\
\hline
\end{tabular}

Tabla 1. Porcentaje de alumnos/as según puntuación en la escala (1-6). 
Presentamos algunos de los verbatim (expresiones literales) más significativos de los estudiantes:

"es el método más adecuado para evaluar la comunicación"

"el examen escrito no habría sido un método adecuado para evaluar habilidades"

"no me fío de la nota de mis compañeros", "prefiero que me califique el profesor";

"evaluar a los compañeros me ayuda en mi propia escenificación", "se distinguen muy bien desde fuera las diversas técnicas de comunicación", "observar cómo lo hacen otros es un buen aprendizaje"

"en una situación real yo lo haría mejor".

En cuanto a la concordancia entre la puntuación obtenida entre los estudiantes y los profesores, en la tabla 2 se muestran las medianas y amplitud intercuartil de la puntuación de estudiantes y profesores respecto a cada uno de los cuatro bloques definidos en la rúbrica, y la puntuación total.

\begin{tabular}{|c|c|c|c|c|c|}
\hline & $\begin{array}{l}\text { Comp. } \\
\text { No verbal }\end{array}$ & $\begin{array}{c}\text { Comp. } \\
\text { Paraverbal }\end{array}$ & $\begin{array}{l}\text { Relación } \\
\text { de Ayuda }\end{array}$ & $\begin{array}{l}\text { Técn. } \\
\text { Comunicación }\end{array}$ & TOTAL \\
\hline Estudiante & 16 (15 a 16$)$ & 12 (12 a 12$)$ & $4(3$ a 4) & 26 (21 a 28$)$ & $\begin{array}{c}56(51 \mathrm{a} \\
60)\end{array}$ \\
\hline Profesor & 12 (11 a 13$)$ & 10 (8 a 10) & 3 (2 a 3) & 20 (16 a 22$)$ & $\begin{array}{c}43(39 a \\
47)\end{array}$ \\
\hline $\begin{array}{c}\text { Máxima } \\
\text { puntuación }\end{array}$ & 16 & 12 & 4 & 28 & 60 \\
\hline $\mathrm{CCl}$ & $\begin{array}{c}0,12 \\
(0 \mathrm{a} 0,36)\end{array}$ & $\begin{array}{c}0,12 \\
(0 \text { a } 0,9)\end{array}$ & $\begin{array}{c}0,16 \\
(0 \text { a } 0,4)\end{array}$ & $\begin{array}{c}0,17 \\
(0 \text { a } 0,37)\end{array}$ & $\begin{array}{c}0,09 \\
(0 \text { a } 0,27)\end{array}$ \\
\hline
\end{tabular}

Tabla 2.- Comparación y concordancia de puntuaciones entre estudiantes y profesores.- 
El grado de adquisición de habilidades en cada uno de los bloques relacionados con componentes de la comunicación y relación de ayuda, en opinión de los profesores es el siguiente:

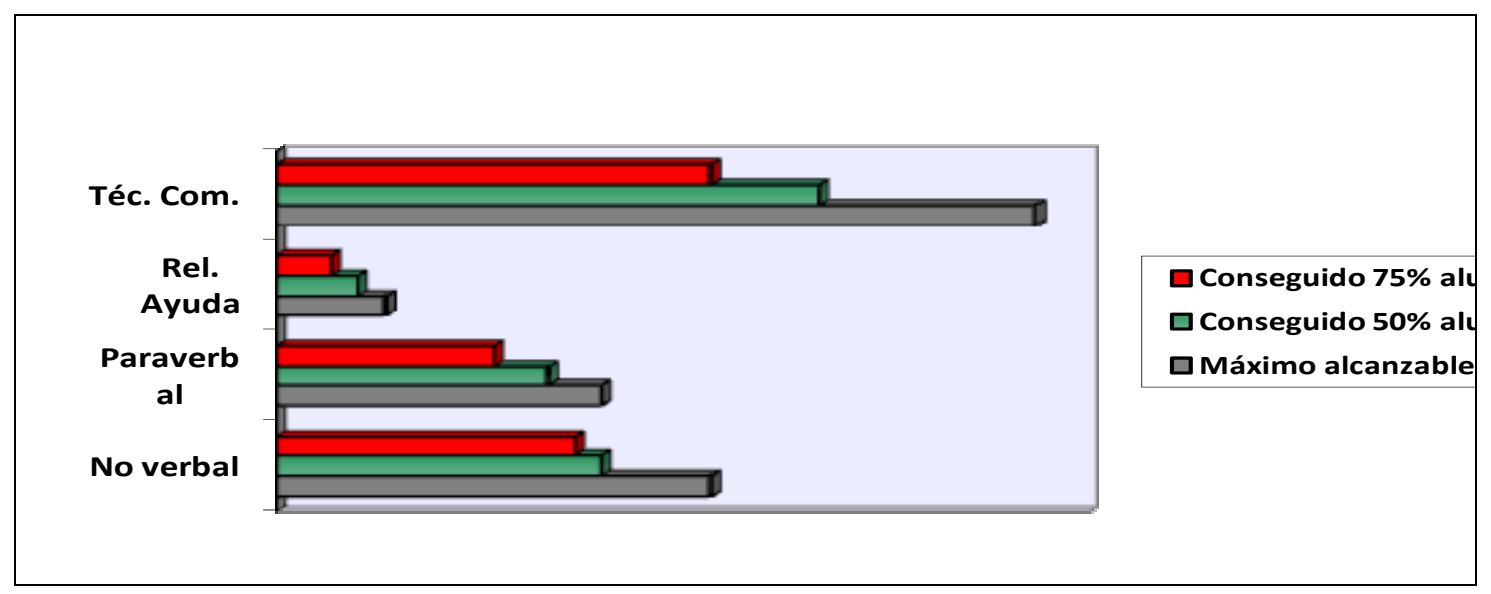

Gráfico 1.- Adquisición de habilidades por componentes de la Comunicación.-

\section{DISCUSIÓN Y CONCLUSIONES}

En relación a la escenificación como método de aprendizaje y evaluación, tanto los resultados cuantitativos como los comentarios realizados muestran un alto grado de satisfacción de los estudiantes. El 90\% de los estudiantes manifiesta una opinión positiva sobre el método de evaluación (3 o más sobre 6), y la mediana es 5 . Los verbatim recogidos corroboran estos resultados "es el método más adecuado para evaluar la comunicación".

No hemos encontrado estudios de características similares con los que comparar nuestros resultados. Sin embargo, a la vista de los mismos, consideramos que la escenificación como método de aprendizaje y evaluación es una herramienta aceptada en forma general por los alumnos.

En cuanto a la concordancia entre la evaluación realizada por estudiantes y profesores encontramos que es "insignificante" (menor de 0,2) (Landis y Koch, 1977). Hemos podido comprobar que esta falta de concordancia, que en principio podría ser preocupante, aparece igualmente en estudios donde se compara la evaluación realizada por distintos profesores. Otros estudios, como el de Warren Piper, apuntan que existe mucha confusión entre los examinadores (estudiantes y profesores) acerca de la aplicación de los criterios de evaluación (Brown y Glasner, 2007). Aún así, se nos abre una ventana de oportunidad para la mejora de la concordancia. Aspectos como la clarificación y la negociación previa con los estudiantes de los criterios de evaluación, la revisión de estructuras y contenidos de las escenificaciones y la posibilidad de introducir filmaciones, tanto para el aprendizaje como la evaluación, son elementos de mejora a planificar en próximos cursos.

Sobre la utilidad de las escenificaciones para la adquisición de competencias en habilidades comunicativas, parece que es eficaz para esta materia, y pensamos que es exportable a otro tipo de entornos y materias relacionadas con habilidades. 
Respecto a la dimensión no verbal de la comunicación, el nivel mínimo alcanzado por el $75 \%$ de los estudiantes se traduce en un patrón y frecuencia en la mirada agradables, una postura abierta que demuestra aceptación, una distancia oportuna y dinámica gestual buena. En relación con la dimensión paraverbal, se manifiesta que el volumen de voz es adecuado, sin apenas perturbaciones o pausas desagradables y la velocidad es apropiada. En cuanto a las fases de relación de ayuda, se demuestra que conocen algunas de las fases, pero no las aplican intencionadamente. Acerca de las técnicas de comunicación, los estudiantes intercalan opiniones y juicios que interrumpen la expresión de los pacientes simulados, utilizan frases de empatía o contacto físico inadecuados, solo utilizan preguntas abiertas o cerradas, paráfrasis o aclaración pero no ambas, hacen resumen de forma errónea, impone al paciente el objetivo/s y comete errores al intentar utilizar técnicas de asertividad. Por otra parte, el nivel mínimo alcanzado por el $50 \%$ de los alumnos/as, en los apartados de relación de ayuda y técnicas de comunicación, presenta una adquisición de habilidades mucho más favorable; como por ejemplo aplicar las fases de la relación de ayuda con pequeños errores, dejar hablar al paciente, utilizar preguntas abiertas y cerradas.

Existen estudios experimentales que ofrecen ciertas pruebas de que el entrenamiento en habilidades comunicativas en profesionales de la salud (enfermeras y médicos) puede tener un efecto beneficioso en la conducta comunicativa. Estas mejoras no se detectaron en los grupos sin intervención o con comentarios o críticas únicamente (Fallowfield, 2002 y Razavi, 2002).

Razavi señala que el grupo de enfermeras con entrenamiento tuvo más dominio de la entrevista. Además usaron palabras significativamente diferentes en lo que respecta al contenido emocional. El tipo de entrenamiento que demuestra eficacia es el entrenamiento "cara a cara". Sería de interés investigar los resultados en términos de salud que produce en los pacientes el aumento de la calidad en la comunicación de los profesionales que les atienden.

La evaluación de la aplicación del conocimiento a casos reales simulados, es una práctica de evaluación innovadora. Esto equivale a dirigir la evaluación, a analizar en qué medida los estudiantes son capaces de aplicar el conocimiento para resolver problemas de la vida real y tomar las decisiones adecuadas. Glaser ya hacía referencia en 1990, a la importancia de la evaluación del "conocimiento utilizable" (Dochy, 2002). Este estudio es un ejemplo de ello. 


\section{REFERENCIAS BIBLIOGRÁFICAS}

Brown, S. y Glasner, A. (2007). Evaluar en la universidad. Problemas y nuevos enfoques. Madrid: Narcea.

Caballer, A., Oliver, M.J. y Gil, J.M. (2005). Escala de Expresión/Comunicación corporal para estudiantes universitarios. Revista de Investigación Educativa, 23 (1), 7-22.

Cléries, X., Borrell, F., Epstein, R.M., Kronfly, E. y Escoda, J.J. (2003). Aspectos comunicacionales: el reto de la competencia de la profesión médica. Atención Primaria, 32(2),110-7.

Dochy, F., Seger, M. y Dierick, S. (2002). Nuevas Vías de Aprendizaje y Enseñanza y sus Consecuencias: una Nueva Era de Evaluación. Revista de Docencia Universitaria, 2 (2), 13-29.

Fallowfield, L., Jenkins, V., Farewell, V., Saul J., Duffy A. y Eves R. (2002) Efficacy of a Cancer Research UK communication skills training model for oncologists: a randomised controlled study. Lancet, 359(9307),650-6. En: La Biblioteca Cochrane Plus, 2008 Número 2. Oxford: Update Software Ltd. Recuperado de: http://www.update-software.com. (Traducida de The Cochrane Library, 2008 Issue 2. Chichester, UK: John Wiley \& Sons, Ltd.).

Fellowes, D., Wilkinson, S. y Moore, P. (2008). Entrenamiento en habilidades comunicativas para los profesionales de la asistencia sanitaria que trabajan con pacientes con cáncer, sus familias o cuidadores (Revisión Cochrane traducida). En: La Biblioteca Cochrane Plus, 2008 Número 2. Oxford: Update Software Ltd. Recuperado de: http://www.update-software.com. (Traducida de The Cochrane Library, 2008 Issue 2. Chichester, UK: John Wiley \& Sons, Ltd.).

Fernández, E. (2005). Competencias clínicas en Enfermería: modelos de aprendizaje y de gestión de la práctica. Educare21, 21. Recuperado de: http://www.enfermeria21.com/educare

Flecha, R. y Tortajada, I. (2002). Retos y salidas educativas en la entrada de siglo. In F. Imbernon, et al. (Eds.), La educación en el siglo XXI los retos del futuro inmediato. (4a ed., pp. 13). Barcelona: Graó.

Gobierno de Navarra. Sección de Promoción de la Salud. Instituto de Salud Pública (2006). Manual de Educación para la Salud.

Landis, J.R. y Koch, G.G. (1977). The measurement of observer agreement for categorical data. Biometrics, 33, 159-174.

López, I. y González, J.L. y Agudo E. (2007). Desarrollo de Competencias Transversales a través del método ABP en la asignatura Enfermería Comunitaria II. Comunicación presentada en IV Jornadas Internacionales de Innovación Universitaria de la Universidad Europea de Madrid. Julio 2007.

Marriner, A. (2007): Modelos y Teorías de Enfermería. 6a ed. Elsevier España SA.

Mejía, M.E. (2006). Reflexiones sobre la relación interpersonal enfermera-paciente en el ámbito del cuidado clínico. Index de Enfermería, 15 (54), 48-52. Accesible en Google Académico. 
Razavi, D., Delvaux, N., Marchal, S., Durieux, J.F, Farvacques, C., Dubus, L. et al.(2002). Does training increase the use of more emotionally laden words by nurses when talking with cancer patients? A randomised study. British Journal of Cancer, 87, 1-7. included - new for update, not in original searches. En: La Biblioteca Cochrane Plus, 2008 Número 2. Oxford: Update Software Ltd. Recuperado de: http://www.update-software.com (Traducida de The Cochrane Library, 2008 Issue 2. Chichester, UK: John Wiley \& Sons, Ltd.).

Universidad Europea Madrid (2008). Plan de Estudios de Grado en Enfermería en la Universidad Europea de Madrid: Asignatura "Habilidades para Comunicar y Educar en Salud".

Zabalza, M.A. (2007). Competencias docentes del profesorado universitario. Calidad y desarrollo profesional. Madrid: Narcea S.A. 


\section{ANEXO}

Rúbrica con los ítems y escala para estimar el grado de consecución de los objetivos y competencias

\begin{tabular}{|l|l|l|l|l|}
\hline \multicolumn{1}{|c|}{ Ítem a evaluar } & 1 & 2 & 3 & 4 \\
\hline BLOQUE 1 COMPONENTE NO VERBAL & & & & \\
\hline Mirada & & & & \\
\hline Postura & & & & \\
\hline Gestos & & & & \\
\hline Distancia & & & & \\
\hline BLOQUE 2 COMPONENTE PARAVERBAL & & & & \\
\hline Volumen & & & & \\
\hline Fluidez & & & & \\
\hline Velocidad & & & & \\
\hline & & & & \\
\hline BLOQUE 3 RELACION DE AYUDA & & & & \\
\hline Conocimiento de las fases & & & & \\
\hline BLOQUE 4 TECNICAS DE COMUNICACIÓN & & & & \\
\hline Escucha activa & & & & \\
\hline Empatía & & & & \\
\hline Preguntas abiertas y cerradas & & & & \\
\hline Técnicas de feed-back (paráfrasis y aclaración) & & & & \\
\hline Resumen & & & & \\
\hline Técnicas de negociación & & & & \\
\hline Técnicas de asertividad & & & & \\
\hline
\end{tabular}

\section{MIRADA}

1. Mira muy poco. Impresión negativa. Mira continuamente. Muy desagradable.

2. Mira poco. Impresión algo negativa. Mira en exceso. Desagradable.

3. Frecuencia y patrón de mirada buenos. Agradable.

4. Frecuencia y patrón de mirada muy buenos. Muy agradable.

\section{POSTURAS}

1. Postura muy cerrada. Da la impresión de un rechazo total.

2. Postura algo cerrada. Da la impresión de un rechazo parcial.

3. Postura abierta. Da la impresión de aceptación.

4. Postura bastante abierta. Da la impresión de una gran aceptación. 


\section{DISTANCIA / CONTACTO FÍSICO}

1. Distancia excesiva o extremadamente próxima e íntima. Muy desagradable.

2. Distancia demasiado próxima para una interacción casual. Desagradable.

3. Distancia oportuna. Impresión de acercamiento. Agradable.

4. Distancia excelente. Buena impresión de acercamiento. Muy agradable.

\section{GESTOS}

1. No hace ningún gesto, manos inmóviles. Impresión muy negativa.

2. Algunos gestos pero escasos. Impresión negativa.

3. Buena frecuencia y distribución de los gestos. Impresión positiva.

4. Muy buena frecuencia y distribución de los gestos. Impresión muy positiva.

\section{VOLUMEN DE LA VOZ}

1. Volumen excesivamente bajo o alto (casi llega al grito).Muy desagradable.

2. Se le oye ligeramente. Voz baja o demasiado alto. Impresión algo negativa.

Desagradable.

3. Volumen de voz bastante adecuado. Impresión positiva.

4. Volumen de voz muy adecuado. Impresión muy positiva.

\section{FLUIDEZ}

1. Muchas perturbaciones o muchas pausas embarazosas. Muy desagradable.

2. Frecuentes perturbaciones o pausas embarazosas. Desagradable.

3. Sin apenas perturbaciones y pausas embarazosas. Agradable.

4. Sin perturbaciones ni pausas embarazosas. Muy agradable.

\section{VELOCIDAD}

1. Habla extremadamente deprisa o despacio. No se le entiende nada. Muy desagradable.

2. Habla bastante deprisa o despacio. A veces no se le entiende. Desagradable.

3. Velocidad de habla bastante apropiada. Agradable.

4. Velocidad de habla muy apropiada. Muy agradable.

\section{CONOCIMIENTO DE LAS FASES DE RELACIÓN DE AYUDA}

1. En la entrevista se evidencia que no conoce las fases de la relación de ayuda.

2. Conoce alguna de las fases.

3. Conoce las fases y las aplica con algún error (desordenadas, saltos entre ellas).

4. Conoce las fases y las aplica secuencialmente sin errores.

\section{ESCUCHA ACTIVA}

1. No deja expresarse al paciente, interrumpe constantemente, juzga, opina.

2. Deja hablar al paciente pero intercala opiniones y juicios que interrumpen la expresión.

3. Deja hablar al paciente. Alguna interrupción ocasional muy aislada.

4. Deja hablar al paciente, tiene actitud de escucha. 


\section{EMPATIA}

1. No demuestra empatía en ningún momento.

2. Utiliza frases de empatía o contacto físico inadecuadas en forma y momento.

3. Utiliza frases de empatía o contacto físico adecuadas en forma pero no en momento.

4. Utiliza frases de empatía o contacto físico adecuadas en forma y momento.

\section{PREGUNTAS ABIERTAS Y CERRADAS}

1. No utiliza preguntas.

2. Solo utiliza preguntas abiertas o cerradas.

3. Utiliza preguntas abiertas y cerradas pero alguna vez de de forma inadecuada (momento o forma)

4. Utiliza preguntas abiertas y cerradas de forma adecuada.

\section{TECNICAS DE FEED-BACK}

1. No utiliza aclaración ni paráfrasis en ningún momento.

2. Utiliza aclaración o paráfrasis una vez, pero no ambas. O las utiliza en cada frase, interrumpiendo la comunicación.

3. Utiliza aclaración o paráfrasis más de una vez, pero no ambas.

4. Utiliza aclaración y paráfrasis más de una vez.

\section{RESUMEN}

1. No utiliza el resumen.

2. Utiliza el resumen de forma errónea y un momento no adecuado.

3. Utiliza el resumen en el momento adecuado, con pequeños errores.

4. Utiliza el resumen en el momento adecuado.

\section{TECNICAS DE NEGOCIACIÓN}

1. No utiliza ninguna técnica de negociación

2. Impone al paciente el objetivo o la actividad.

3. Pregunta la opinión del paciente, pero no utiliza técnicas de negociación.

4. Pregunta al paciente y utiliza técnicas de negociación.

\section{TECNICAS DE ASERTIVIDAD}

1. No utiliza ninguna técnica de asertividad.

2. Comete graves errores al intentar utilizar alguna técnica.

3. Utiliza alguna técnica de asertividad, con pequeños errores.

4. Utiliza al menos una técnica de asertividad, de forma correcta 


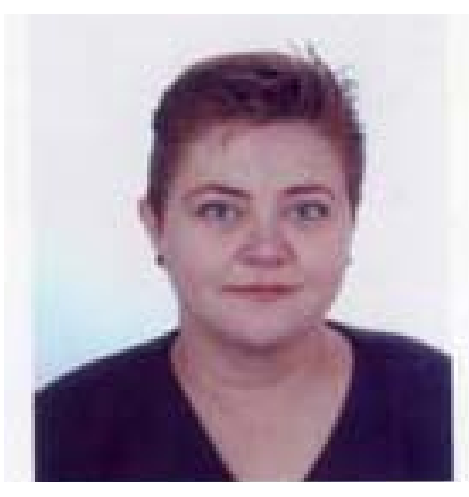

\section{Må ÁNGELES FERNÁNDEZ DÍAZ}

Universidad Europea de Madrid

Departamento de Enfermería

Mail: mariaangeles.fernandez@uem.es

Enfermera. Experta Universitaria en Gestión de Servicios de Enfermería. Máster en Gestión Sociosanitaria.

Enfermera Comunitaria en el Área de Moratalaz (Madrid). Profesora Ayudante a tiempo parcial en el Departamento de Enfermería de la Universidad Europea de Madrid.

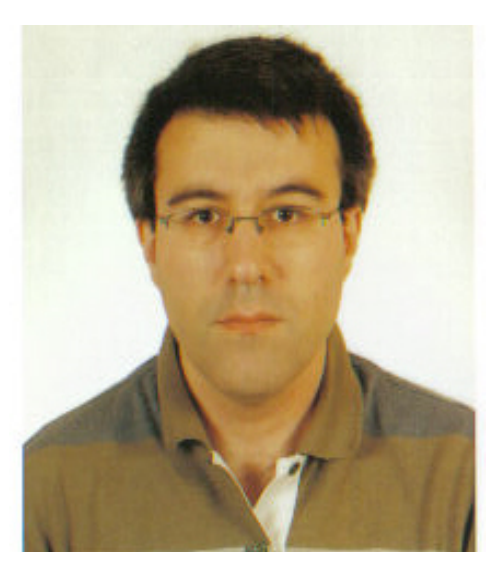

\section{JUAN LUIS GONZÁLEZ PASCUAL}

Universidad Europea de Madrid

Departamento de Enfermería

Mail: juanluis.gonzalez2@uem.es

Enfermero. Antropólogo Social y Cultural. Máster en Epidemiología y Salud Pública. Profesor Ayudante a tiempo completo en el Departamento de Enfermería de la Universidad Europea de Madrid. 


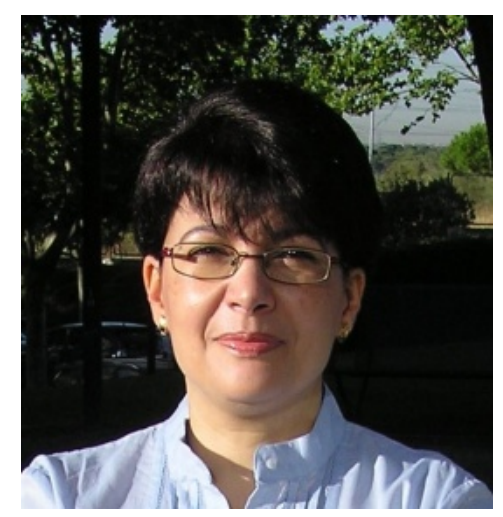

\section{INMACULADA LÓPEZ MARTÍN}

Universidad Europea de Madrid

Departamento de Enfermería

Mail: inmaculada.lopez@uem.es

Enfermera. Licenciada en Humanidades. Máster en Educación y Gestión de Enfermería. Responsable de la Acción Tutorial en Vicerrectorado de Estudiantes y Empleo de la Universidad Europea de Madrid y profesora del Departamento de Enfermería de la Universidad Europea de Madrid.

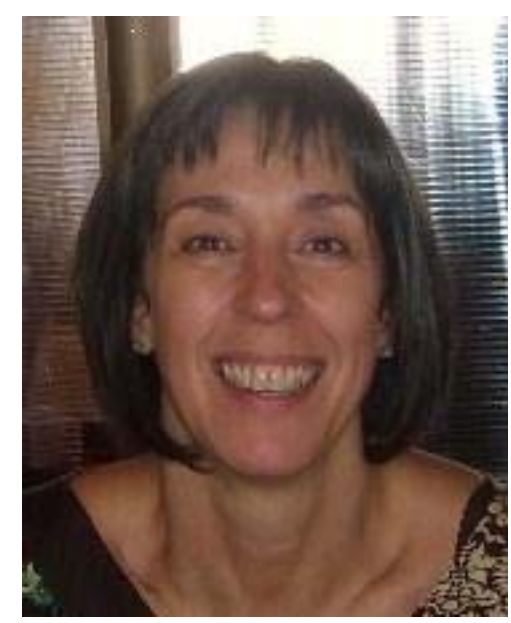

\section{Ma ESPERANZA MANSO MARTÍNEZ \\ Universidad Europea de Madrid \\ Departamento de Enfermería}

E.A.P. Villacastín y El Espinar (Segovia)

Mail: mariaesperanza.manso@uem.es

Enfermera. Psicopedagoga. Especialista en Asistencia Obstétrica.

Profesora Ayudante a tiempo parcial en el Departamento de Enfermería de la Universidad Europea de Madrid. Matrona en los Equipos de Atención Primaria de El Espinar y de Villacastín (Gerencia Atención Primaria de Segovia). Tutora de prácticas de la Unidad Docente de Matronas de Valladolid. 
